\title{
A linear model for the structure of turbulence beneath surface water waves
}

Article

Accepted Version

Teixeira, M. A.C. (2011) A linear model for the structure of turbulence beneath surface water waves. Ocean modelling, 36 (1-2). pp. 149-162. ISSN 1463-5003 doi:

https://doi.org/10.1016/j.ocemod.2010.10.007 Available at https://centaur.reading.ac.uk/29239/

It is advisable to refer to the publisher's version if you intend to cite from the work. See Guidance on citing.

Published version at: http://dx.doi.org/10.1016/j.ocemod.2010.10.007

To link to this article DOI: http://dx.doi.org/10.1016/j.ocemod.2010.10.007

Publisher: Elsevier Inc.

All outputs in CentAUR are protected by Intellectual Property Rights law, including copyright law. Copyright and IPR is retained by the creators or other copyright holders. Terms and conditions for use of this material are defined in the End User Agreement.

\section{www.reading.ac.uk/centaur}

\section{CentAUR}

Central Archive at the University of Reading

Reading's research outputs online 


\title{
A linear model for the structure of turbulence beneath surface water waves
}

\author{
M. A. C. Teixeira* \\ CGUL, IDL, University of Lisbon, Lisbon, Portugal
}

\begin{abstract}
The structure of turbulence in the ocean surface layer is investigated using a simplified semi-analytical model based on rapid-distortion theory. In this model, which is linear with respect to the turbulence, the flow comprises a mean Eulerian shear current, the Stokes drift of an irrotational surface wave, which accounts for the irreversible effect of the waves on the turbulence, and the turbulence itself, whose time evolution is calculated. By analysing the equations of motion used in the model, which are linearised versions of the Craik-Leibovich equations containing a 'vortex force', it is found that a flow including mean shear and a Stokes drift is formally equivalent to a flow including mean shear and rotation. In particular, Craik and Leibovich's condition for the linear instability of the first kind of flow is equivalent to Bradshaw's condition for the linear instability of the second. However, the present study goes beyond linear stability analyses by considering flow disturbances of finite amplitude, which allows calculating turbulence statistics and addressing cases where the linear stability is neutral. Results from the model
\end{abstract}

\footnotetext{
*Corresponding author: M. A. C. Teixeira, Centro de Geofísica da Universidade de Lisboa, Edifício C8, Campo Grande, 1749-016 Lisbon, Portugal

Email addresses: mateixeira@fc.ul.pt (M. A. C. Teixeira)
} 
show that the turbulence displays a structure with a continuous variation of the anisotropy and elongation, ranging from streaky structures, for distortion by shear only, to streamwise vortices resembling Langmuir circulations, for distortion by Stokes drift only. The TKE grows faster for distortion by a shear and a Stokes drift gradient with the same sign (a situation relevant to wind waves), but the turbulence is more isotropic in that case (which is linearly unstable to Langmuir circulations).

Key words: Langmuir circulation, Turbulent boundary layer, Wave drift velocity, Shear flow, Rapid-distortion theory

\section{Introduction}

Turbulent shear flows beneath surface waves in the upper ocean are characterised by a number of intriguing features that need to be better understood. For example, the turbulence is often dominated by elongated vortices with their axes of rotation aligned in the streamwise direction, i.e. the direction of the wind stress and of wave propagation (Faller and Auer, 1988). These vortices have been identified with Langmuir circulations (Leibovich, 1983), prompting McWilliams et al. (1997) to coin the term 'Langmuir turbulence'. The structure of Langmuir turbulence contrasts with the structure of turbulence typical of boundary layers near flat boundaries, where the flow is dominated by 'streaky structures' (Kline et al., 1967), which are elongated fluctuations of the streamwise velocity component. Thais and Magnaudet's (1996) laboratory experiments of turbulence beneath surface waves sheared by the wind have shown that, even when there are no Langmuir circulations as usually defined, the turbulence is more isotropic than expected near a flat 
boundary and the TKE level is higher.

Langmuir circulations have been explained by Craik and Leibovich (1976), Craik (1977) and Leibovich (1980) as being due to an instability associated with the interaction between the shear current induced by the wind and the Stokes drift of surface waves, but in these studies turbulence was ignored, except as a diffusive process. In a series of papers, Craik (1982), Phillips and $\mathrm{Wu}$ (1994) and Phillips et al. (1996) investigated theoretically the instability of flows with strong shear near wavy boundaries to streamwise vortices. These studies have shown that the basic factors affecting the stability of the flow to perturbations are the shape of the mean velocity profile and the shape of the pseudo-momentum profile (which replaces the Stokes drift as a key parameter for strongly sheared flows) (Andrews and McIntyre, 1978).

Craik and Leibovich's and Phillips' studies treat the generation of streamwise vortices essentially from a linear stability analysis perspective. A theoretical understanding of Langmuir circulations at stages subsequent to instability, when fully developed turbulence exists, requires the use of statistical methods. A first attempt at tackling this problem was made by Teixeira and Belcher (2002). Using an idealised linear model based on rapid distortion theory (RDT), they found that initially isotropic turbulence distorted by a surface wave becomes anisotropic in a way consistent with the existence of intense and elongated streamwise vortices. This process is associated with the transfer of energy from the waves to the turbulence, which leads to a decay of the waves. This wave decay was evaluated using real ocean data by Ardhuin and Jenkins (2006) and Kantha et al. (2009). As in Craik and Leibovich's theory, the Stokes drift of the wave is essential in the process studied 
by Teixeira and Belcher (2002), but acts by distorting the vorticity of the turbulence, so the existence of mean shear is not required. Turbulence acts in this case as the source of the streamwise vortices instead of a dissipative process that limits their growth. According to Teixeira and Belcher (2010), the neglect of shear may be acceptable at late stages in the development of Langmuir circulations, when extensive vertical mixing has taken place, but that is not always the case.

The turbulent shear flow beneath surface waves comprises three basic components: turbulence, a mean Eulerian shear current (induced by the wind stress, by wave breaking, or by viscous boundary layer effects) and the orbital motion of the waves. These three flow components interact in a complex way. The turbulence generally extracts energy from the shear and the wave motion, and the anisotropy of the turbulence is shaped by the distortions caused by the other components of the flow. The distortion by shear, which gives rise to the typical turbulence anisotropy in flat-wall boundary layers, has been studied extensively using RDT (Townsend, 1970; Gartshore et al., 1983; Lee et al., 1990), but the distortion by the wave motion is more subtle and less well understood. This study aims to extend the RDT model of Teixeira and Belcher (2002) to include the distorting effect of shear, thereby approximating better the dynamics of real boundary layers. As in Teixeira and Belcher (2002), the Stokes drift of surface waves will be treated as equivalent to a purely irrotational straining.

As noted by Shrira (1993), when surface waves propagate in a homogeneous fluid with a non-uniform velocity profile, there is only one situation where the wave motion remains irrotational: when the shear rate is con- 
stant and the shear is aligned with the wavenumber vector. Only in that case can the Stokes drift gradient be considered as strictly equivalent to an irrotational straining. Generally, this is not so. The existence of either curvature in the mean velocity profile or an angle between the mean shear and the wavenumber vector renders the wave motion (and the associated Stokes drift) rotational. This makes the dynamics of the motion more complicated and requires the introduction of a generalised Stokes drift with non-zero vorticity (Phillips, 2001). This problem is especially acute in the atmosphere (Phillips and $\mathrm{Wu}, 1994$ ). In the ocean, the waves are observed to be approximately irrotational, perhaps due to the relative weakness of shear currents, and this is taken here as an indication that the adoption of an irrotational Stokes drift is acceptable. Incidentally, this is also the approach used in numerous large-eddy simulation (LES) studies of the oceanic boundary layer (McWilliams et al., 1997; Li et al., 2005; Polton and Belcher, 2007; Grant and Belcher, 2009), when they adopt equations including Craik and Leibovich's 'vortex force', which assumes irrotational wave motion. This provides some reassurance that the present approach is sound.

In this study, the simplest situation of shear-Stokes drift-turbulence interaction is considered: a flow where the shear of the mean Eulerian current and the Stokes drift are aligned and where the gradients of both transports are approximated as (at least locally) constant (cf. Teixeira and Belcher, 2010). As seen above, this approach is self-consistent, and the Stokes drift gradient can be viewed simply as a vertical velocity gradient which has no vorticity associated.

This paper is organised as follows. Section 2 discusses the theoretical 
model used in this study. In Section 3, various statistics of the turbulence are presented for different relative magnitudes of shear and Stokes drift. That section contains an analysis of the energy budget of the turbulence and a physical interpretation of the results. Qualitative and quantitative comparisons of the results with available data are also carried out. Finally, Section 4 presents the main conclusions of this study.

\section{Turbulence distorted by shear and a Stokes drift}

Like all models based on RDT, the present model is linearised with respect to the turbulence (Batchelor and Proudman, 1954). Formally, this linearisation is approximately valid if the strain rate imposed on the turbulence by the shear flow and the Stokes drift is considerably larger than the inverse of the eddy turn-over time and if the time since the beginning of the distortion is smaller than one eddy turn-over time. These conditions have been extensively discussed elsewhere (Hunt and Carruthers, 1990; Kevlahan and Hunt, 1997). Even when these conditions are not strictly satisfied, RDT is useful because it gives the evolution of the turbulence due to external forcings, and previous studies suggest that these forcings are essentially what determines the equilibrium structure of the turbulence (Townsend, 1970; Lee et al., 1990; Mann, 1994; Cambon and Scott, 1999).

Although RDT is closely related to linear stability theory, as pointed out by Speziale et al. (1996) for rotating shear flows, it is more complete in a number of ways. Firstly, it considers perturbations of a finite amplitude. Secondly, it does not impose an exponential dependence on the time evolution of these perturbations (Hunt and Carruthers, 1990). The special cases 
of turbulence distortion by shear only, treated by Townsend (1976), and by Stokes drift only, treated by Teixeira and Belcher (2002, 2010), are quite relevant physically, but they are linearly neutral because the perturbation energy grows at an algebraic rate. Thirdly, unlike linear stability analysis, RDT considers a spectrum of perturbations. This is much more appropriate for describing the structure of real flows, where the turbulence contains a variety of scales, and Reynolds stresses and dissipation rates are the measured or diagnosed quantities (see for example Thais and Magnaudet, 1996; McWilliams et al., 1997, Teixeira and Belcher, 2000).

The momentum equation adopted in this study can be expressed as

$$
\frac{\partial \mathbf{u}}{\partial t}+\mathbf{U} \cdot \nabla \mathbf{u}+\mathbf{u} \cdot \nabla \mathbf{U}=-\frac{1}{\rho} \nabla p^{\prime}+\mathbf{U}_{S} \times \omega
$$

where $\mathbf{u}$ is the turbulent velocity, $\omega=\nabla \times \mathbf{u}$ is the turbulent vorticity, $\mathbf{U}$ is the mean Eulerian velocity, $\mathbf{U}_{S}$ is the Stokes drift velocity, $\rho$ is the density (assumed to be constant) and

$$
p^{\prime}=p+\rho \mathbf{U}_{S} \cdot \mathbf{u}
$$

is a turbulent pressure that includes a correction due to the Stokes drift, as in equation (2.1) of McWilliams et al. (1997). Note that (1) is a linearised and inviscid version of the Craik-Leibovich momentum equation (Leibovich, 1983). Gravity does not appear in this equation because it is assumed to be balanced exactly by the mean pressure in the bulk of the fluid through hydrostatic equilibrium. The last term on the right is the 'vortex force' term introduced to parameterise the Lagrangian-mean effect of the Stokes drift. The corrected pressure $p^{\prime}$ is also a linearised version of the corresponding quantity (called $\pi$ ) used by McWilliams et al. (1997). The Craik-Leibovich 
momentum equation, from which (1) results, can be derived through suitable averaging of the fundamental equations including the wave motion (Craik and Leibovich, 1976), or directly from the basic generalised-Lagrangian-mean equations (Andrews and McIntyre, 1978) assuming that the flow perturbations are irrotational (see also Ardhuin et al., 2008). This approach is not incompatible with the simulation of turbulent flows, since in the momentum equation the turbulent velocity may be grouped in with the rotational mean flow. This is in fact consistent with the RDT assumptions, since the turbulent flow is assumed to be much slower than the wave orbital motions and hence can be considered itself a mean flow from the viewpoint of averaging with respect to the wave oscillations.

The mass conservation equation for the turbulence, valid for incompressible flow,

$$
\nabla \cdot \mathbf{u}=0
$$

completes the set from which $\mathbf{u}$ and $p^{\prime}$ can be determined. An alternative equation set can be formed with (1) and the equation obtained by taking the divergence of (1) and using (3):

$$
\nabla^{2} p^{\prime}=\rho\left\{\nabla \cdot\left[\left(\mathbf{U}+\mathbf{U}_{S}\right) \times \omega\right]+\nabla \cdot[\mathbf{u} \times(\nabla \times \mathbf{U})]-\nabla^{2}(\mathbf{U} \cdot \mathbf{u})\right\}
$$

which gives the corrected pressure.

For simplicity, in the present model both $\mathbf{U}$ and $\mathbf{U}_{S}$ are assumed to depend only on $z$ (the vertical coordinate) and $\mathbf{U}_{S}$ is assumed to be aligned in the $x$ (hereafter called the streamwise) direction. $\mathbf{U}$ may not be aligned in the $x$ direction, but only its $x$ component $U$ is assumed to vary with $z$. The component of the Eulerian flow along $y$ (hereafter called the spanwise 
direction), $V$, is dynamically irrelevant, since it is assumed to be constant, and can be eliminated through a simple Galilean transformation. The possibility $V \neq 0$ is contemplated because typically the Eulerian current existing at the water surface is misaligned with the wind stress due to Ekman layer effects (Ardhuin et al., 2009). However, the vertical gradient of this current (which is proportional to the wind stress) is approximately aligned with the the wavenumber vector of the dominant surface waves, and therefore with their Stokes drift, at least near the surface. The effect of the Earth's rotation on the turbulent motion is approximately negligible, as shown by Grant and Belcher (2009). All of these aspects are consistent with the assumptions of the model.

A Lagrangian mean velocity may be defined as

$$
\mathbf{U}_{L}(z)=\mathbf{U}(z)+\mathbf{U}_{S}(z)
$$

This is the velocity at which fluid parcels are advected on average. It is also possible to define a parameter $\alpha$ such that

$$
\left.\begin{array}{l}
\frac{d U}{d z}=\alpha \frac{d U_{L}}{d z}, \\
\frac{d U_{S}}{d z}=(1-\alpha) \frac{d U_{L}}{d z} .
\end{array}\right\}
$$

This parameter controls the relative importance of the distortion by shear and by Stokes drift. When $\alpha=0$, the distorting mean flow has no vorticity, i.e. it is an irrotational Stokes drift, whereas when $\alpha=1$, the distorting mean flow is a pure shear flow. For values of $\alpha$ between 0 and 1 , the vertical gradients the Eulerian shear flow and of the Stokes drift have the same sign. Outside this interval, the gradients of the Stokes drift and of the shear flow are of opposite signs (see Fig. 1). The case $0<\alpha<1$ is applicable, for 
example, to wind waves, whereas the cases $\alpha<0$ or $\alpha>1$ may correspond to waves propagating against the wind. The case $\alpha=0$ could apply to situations of swell, or of waves propagating over a well-mixed surface layer.

As is usual in RDT, the mean transport gradients (associated with the shear flow and with the Stokes drift) are assumed to be slowly varying, and the turbulence is assumed to be locally homogeneous. Then, at each point the turbulent velocity and pressure can be expressed as Fourier integrals,

$$
\left.\begin{array}{l}
\mathbf{u}(\mathbf{x}, t)=\iiint \hat{\mathbf{u}}(\mathbf{k}, t) \mathrm{e}^{\mathrm{i} \mathbf{k} \cdot \mathbf{x}} \mathrm{d} k_{1} \mathrm{~d} k_{2} \mathrm{~d} k_{3}, \\
p^{\prime}(\mathbf{x}, t)=\iiint \hat{p}^{\prime}(\mathbf{k}, t) \mathrm{e}^{\mathrm{i} \mathbf{k} \cdot \mathbf{x}} \mathrm{d} k_{1} \mathrm{~d} k_{2} \mathrm{~d} k_{3},
\end{array}\right\}
$$

where $\hat{\mathbf{u}}$ and $\hat{p}^{\prime}$ are the corresponding Fourier transforms and $\mathbf{k}(t)=\left(k_{1}, k_{2}, k_{3}(t)\right)$ is the wavenumber vector (cf. Teixeira and Belcher, 2010). The fact that $\mathbf{k}$ depends on time is a consequence of the distortion of the turbulence by the mean transport gradients.

Introducing the definitions of $\mathbf{u}$ and $p^{\prime}$ given by (7) into (1) and (4), equations may be derived for the Fourier transforms of the turbulent velocity and pressure. The Fourier transform of (4) provides a definition for $\hat{p}^{\prime}$, which may be used in the Fourier transform of (1) to eliminate the pressure. This is an important advantage of the spectral formulation used in RDT.

Since the turbulence is random, the primary interest here is in the evolution of the turbulent velocity spectra. Equations for these can be derived by multiplying the equations giving the evolution of the Fourier transforms $\hat{u}_{i}$ by the complex conjugate of each Fourier transform, ensemble averaging and noting that

$$
\overline{\hat{u}_{i}^{*}(\mathbf{k}, t) \hat{u}_{j}\left(\mathbf{k}^{\prime}, t\right)}=\Phi_{i j}(\mathbf{k}, t) \delta\left(\mathbf{k}-\mathbf{k}^{\prime}\right), \quad i, j=1,2,3,
$$


by definition, where $\Phi_{i j}$ is the three-dimensional wavenumber spectrum of the turbulent velocity, $\delta$ is the Dirac delta, the asterisk denotes complex conjugate and the overbar denotes ensemble averaging.

From (8) it can be seen that $\Phi_{i j}$, when real, is symmetric. The equations for the six independent components of this tensor are:

$$
\left.\begin{array}{l}
\frac{\partial \Phi_{11}}{\partial t}=2(1-\alpha) \frac{k_{1} k_{3}}{k^{2}} \frac{d U_{L}}{d z} \Phi_{11}-2\left[\alpha-(1+\alpha) \frac{k_{1}^{2}}{k^{2}}\right] \frac{d U_{L}}{d z} \Phi_{13}, \\
\frac{\partial \Phi_{33}}{\partial t}=2(1+\alpha) \frac{k_{1} k_{3}}{k^{2}} \frac{d U_{L}}{d z} \Phi_{33}-2(1-\alpha) \frac{k_{12}^{2}}{k^{2}} \frac{d U_{L}}{d z} \Phi_{13}, \\
\frac{\partial \Phi_{22}}{\partial t}=2 \frac{k_{2}}{k^{2}} \frac{d U_{L}}{d z}\left[(1+\alpha) k_{1} \Phi_{23}+(1-\alpha) k_{3} \Phi_{12}\right] \\
\frac{\partial \Phi_{13}}{\partial t}=2 \frac{k_{1} k_{3}}{k^{2}} \frac{d U_{L}}{d z} \Phi_{13}-\left[\alpha-(1+\alpha) \frac{k_{1}^{2}}{k^{2}}\right] \frac{d U_{L}}{d z} \Phi_{33} \\
-(1-\alpha) \frac{k_{12}^{2}}{k^{2}} \frac{d U_{L}}{d z} \Phi_{11}, \\
\frac{\partial \Phi_{12}}{\partial t}=(1-\alpha) \frac{k_{1} k_{3}}{k^{2}} \frac{d U_{L}}{d z} \Phi_{12}-\left[\alpha-(1+\alpha) \frac{k_{1}^{2}}{k^{2}}\right] \frac{d U_{L}}{d z} \Phi_{23} \\
+\frac{k_{2}}{k^{2}} \frac{d U_{L}}{d z}\left[(1+\alpha) k_{1} \Phi_{13}+(1-\alpha) k_{3} \phi_{11}\right], \\
\frac{\partial \Phi_{23}}{\partial t}=(1+\alpha) \frac{k_{1} k_{3}}{k^{2}} \frac{d U_{L}}{d z} \Phi_{23}-(1-\alpha) \frac{k_{12}^{2}}{k^{2}} \frac{d U_{L}}{d z} \Phi_{12} \\
+\frac{k_{2}}{k^{2}} \frac{d U_{L}}{d z}\left[(1+\alpha) k_{1} \Phi_{33}+(1-\alpha) k_{3} \Phi_{13}\right] .
\end{array}\right\}
$$

Note that these equations are expressed in terms of $d U_{L} / d z$ and $\alpha$, showing that the vertical gradient of the total Lagrangian mean velocity is responsible for the distortion of the turbulence. A natural dimensionless time suggested by the equations is

$$
\beta=\frac{d U_{L}}{d z} t
$$

One additional equation must be added to (9) to give the evolution of the wavenumber vector due to the distortion by the mean flow:

$$
k_{3}=k_{30}-k_{1} \beta
$$


where $k_{30}=k_{3}(t=0)$ is the initial value of the vertical component of the wavenumber. Note that also this equation depends on the vertical gradient of the Lagrangian velocity, through $\beta$. The other components $\left(k_{1}\right.$ and $\left.k_{2}\right)$ are unaffected by the transport gradients and remain unchanged.

This study aims to investigate the structure of the turbulence, and for that purpose correlations and integral length scales of the turbulent velocity will be calculated. At any time, the velocity variances or one-point correlations are given by the expression

$$
\overline{u_{i} u_{j}}=\iiint \Phi_{i j} \mathrm{~d} k_{1} \mathrm{~d} k_{2} \mathrm{~d} k_{3},
$$

where $i, j=1,2,3$, while the integral length scales are given by

$$
L_{i j}^{x}=\pi \frac{\iint \Phi_{i j}\left(k_{1}=0\right) \mathrm{d} k_{2} \mathrm{~d} k_{3}}{\overline{u_{i} u_{j}}}
$$

along the $x$ direction, with analogous definitions for the $y$ direction. The velocity variances and correlations give information about the turbulence energy and anisotropy. The integral length scales give information about the distances over which the turbulence velocities lose their coherence, and can be used to characterise the turbulence elongation.

In line with previous RDT treatments (Townsend, 1970; Mann, 1994; Teixeira and Belcher, 2002, 2010), the turbulence is assumed to be initially isotropic, so that

$$
\overline{u_{i} u_{j}}(t=0)=q^{2} \delta_{i j},
$$

where $q$ is the initial root-mean-square (RMS) velocity and $\delta_{i j}$ is the Kronecker delta. If $l$ is defined as the initial longitudinal integral length scale, then

$$
L_{i i}^{x_{m}}(t=0)=l \quad \text { if } \quad i=m, \quad L_{i i}^{x_{m}}(t=0)=0.5 l \quad \text { if } \quad i \neq m,
$$


because the longitudinal length scales are twice the transverse length scales in isotropic turbulence.

The time evolution of the variances, correlations and integral length scales is determined in general by solving numerically the equation set (9) for each wavenumber and integrating $\Phi_{i j}$ over all wavenumbers, in accordance with (12) and (13). The solution of the equation set is analytical for some special cases, for example when $\alpha=0$ or $\alpha=1$. When the integrals in (12) and (13) are expressed in polar coordinates, it turns out that the integration over all wavenumber values does not have to be carried out, and it is sufficient to calculate the integrals over all wavenumber directions. This shortens the computation time considerably, since the necessary integrals are two-dimensional for the correlations and variances and one-dimensional for the integral length scales. Also, the behaviour of these quantities does not depend on the spectral form of the initial turbulence, which in fact does not have to be specified (cf. Batchelor and Proudman, 1954; Savill, 1987) (This property would not hold if viscosity had been included).

In this model, $\alpha$ and $\beta$ are the key parameters that control the behaviour of the turbulence statistics. $\beta$, the dimensionless time given by (10), quantifies the total distortion imposed on the turbulence by the shear and the Stokes drift since isotropy. $\alpha$ controls the relative importance of these two distorting effects.

\section{Results: statistics of the turbulence}

Correlations and integral length scales of the turbulent velocity are presented next. Related results for some particular cases have been obtained 
by other authors for turbulence in a rotating shear flow (Speziale and MacGiolla Mhuiris, 1989; Cambon et al., 1994; Salhi and Cambon, 1997), which as will be seen is related to the present flow, but the emphasis was mainly on the evolution of the TKE. Here the parameter regime will be explored more systematically and attention will also be focused on measures of the turbulence anisotropy and turbulence structure (only briefly treated by Salhi and Cambon, 1997). Moreover, in contrast with these previous studies, the results will be interpreted having in mind their application to the oceanic boundary layer.

Figs. 2 to 5 show variances, correlations and integral length scales of the turbulent velocity as a function of the dimensionless time $\beta$, and of parameter $\alpha$. A maximum value of $\beta$ of 10 was chosen, because it is unlikely that the turbulence will undergo distortions greater than this, the anisotropy becoming too large and being limited by nonlinear processes (cf. Townsend, 1976; Lee et al., 1990). The limits of $\alpha$ have been chosen as -1 and 2 , so that the distortion by a Stokes drift gradient and a shear having the same sign (the situation considered by Craik and Leibovich) occupies the central portion of the graphs $(0<\alpha<1)$, but the parameter regime where they have opposite signs $(-1<\alpha<0$ or $1<\alpha<2)$ is also considered (see Fig. 1). It must be stressed that the results of Sections 3.1 to 3.4 are rather general, and do not yet correspond to a particular oceanic depth. A more specific application will be given in Section 3.7.

\subsection{Reynolds stresses}

Fig. 2 shows various components of the Reynolds stress tensor normalised by the initial RMS velocity of the turbulence, $q$. 
Figs. 2a-c show the streamwise, spanwise and vertical velocity variances, respectively $\overline{u^{2}}, \overline{v^{2}}$ and $\overline{w^{2}}$. Fig. 2d shows the TKE, which is defined as $K=(1 / 2)\left(\overline{u^{2}}+\overline{v^{2}}+\overline{w^{2}}\right)$, and Fig. 2e shows the shear stress, $\overline{u w}$. All velocity variances depart from a normalised value of one and the shear stress departs from a value of zero, corresponding to isotropic turbulence.

When interpreting the graphs, it is useful to note that there is a connection between the shear stress and the growth rate of TKE. For a flow including Stokes drift, the mechanical production term of the TKE equation is (McWilliams et al., 1997)

$$
-\overline{u w} \frac{d U_{L}}{d z}
$$

So the tendency of the TKE is roughly proportional to the shear stress through straining by the Lagrangian-mean flow: a negative shear stress leads to TKE growth while a positive shear stress leads to TKE decay.

In Fig. 2 it can be seen that when $\alpha=0$ then $\overline{u^{2}}$ decreases with $\beta$, while both $\overline{v^{2}}$ and $\overline{w^{2}}$ increase at a similar rate. This leads, at large $\beta$, to a situation where $\overline{u^{2}} \ll\left(\overline{v^{2}} \approx \overline{w^{2}}\right)$, that is, turbulence dominated by intense vortices with their axis of rotation aligned in the streamwise direction (Teixeira and Belcher, 2002). The TKE increases at a moderate (algebraic) rate and $\overline{u w}$ takes negative values.

When $\alpha=1$, then $\overline{u^{2}}$ and $\overline{v^{2}}$ both increase with $\beta$, while $\overline{w^{2}}$ decreases. $\overline{u^{2}}$ increases at a faster rate than $\overline{v^{2}}$, and this leads to an ordering of the variances that becomes $\overline{u^{2}}>\overline{v^{2}}>\overline{w^{2}}$ at large $\beta$. This corresponds to turbulence dominated by streaky structures characterised by intense streamwise velocity fluctuations (as simulated, for example, by Lee et al., 1990). The TKE grows faster than for $\alpha=0$ (but still algebraically), and $\overline{u w}$ is more negative, as 
expected.

When $0<\alpha<1$ (shear and Stokes drift gradient with the same sign), all the velocity variances grow very fast (approximately exponentially). For $\alpha=0.5$ (where the maximum growth rate roughly occurs), $\overline{u^{2}}$ and $\overline{w^{2}}$ grow at a similar rate, which is somewhat larger than the growth rate of $\overline{v^{2}}$. However, the magnitude of the three variance components never differs by a large factor and for that reason the turbulence is more isotropic than for either $\alpha=0$ or $\alpha=1$. Obviously, in this case the TKE grows much faster and $\overline{u w}$ also takes much larger negative values than for $\alpha=0$ or $\alpha=1$.

When $\alpha<0$ or $\alpha>1$, the Stokes drift gradient and the shear have opposite signs. All the velocity variances decay as $\beta$ increases (excepting a short initial period of transient growth near $\alpha=0$ and $\alpha=1$ ), and there is a hint in Figs. 2a,c that the velocity variances also oscillate. This behaviour will be explained in Section 3.5. Naturally, the TKE decays in this case, and $\overline{u w}$ is now generally small and positive, except for relatively low $\beta$ and near $\alpha=0$ or $\alpha=1$.

The rapid growth of the TKE that occurs when $0<\alpha<1$ and the TKE decay when $\alpha<0$ or $\alpha>1$ (see Fig. 2d) is consistent with the instability to Langmuir circulations, since this instability arises in neutrally stratified inviscid flow whenever the shear and the Stokes drift gradient have the same sign: $(d U / d z)\left(d U_{S} / d z\right)>0$ (see e.g. Leibovich, 1977). This corroborates the known result that linear instability is included in the possible modes of growth of disturbances in RDT (Speziale et al., 1996). However, Langmuir circulations have usually been regarded as streamwise vortices. The present results suggest that the only situation where pure streamwise vortices exist 
is in the absence of shear (for $\alpha=0$ ). Hence the structure of Langmuir circulations can be more complex than perceived in previous investigations. An analysis of the turbulence anisotropy and structure will make this point clearer.

\subsection{Tubulence anisotropy}

Detailed information about the turbulence anisotropy can be obtained by calculating ratios of the turbulent velocity variances and correlations. This is done in Fig. 3.

Figs. 3a-c present ratios of the vertical to the streamwise velocity variance, $\overline{w^{2}} / \overline{u^{2}}$, of the vertical to the spanwise velocity variance, $\overline{w^{2}} / \overline{v^{2}}$, and of the spanwise to the streamwise velocity variance, $\overline{v^{2}} / \overline{u^{2}}$, respectively. Given Figs. 3a,b, the information provided by Fig. 3c is partly redundant (since $\overline{v^{2}} / \overline{u^{2}}$ can be determined from $\overline{w^{2}} / \overline{u^{2}}$ and $\overline{w^{2}} / \overline{v^{2}}$ ). Either of these ratios can be used as a measure to distinguish between shear turbulence (dominated by streaky structures) and Langmuir turbulence (dominated by streamwise vortices), since the former is characterised by $\overline{w^{2}} / \overline{u^{2}}<1, \overline{w^{2}} / \overline{v^{2}}<1$ and $\overline{v^{2}} / \overline{u^{2}}<1$ and the latter by $\overline{w^{2}} / \overline{u^{2}}>1, \overline{w^{2}} / \overline{v^{2}} \approx 1$ and $\overline{v^{2}} / \overline{u^{2}}>1$.

It can be seen that, as $\beta$ increases, $\overline{w^{2}} / \overline{u^{2}}$ becomes considerably larger than 1 for $\alpha<0.5$, and smaller than 1 for $\alpha>0.5$. The highest and the lowest values of $\overline{w^{2}} / \overline{u^{2}}$ are attained, respectively, for $\alpha=0$ and near $\alpha=1$ (slightly above). The region where $\overline{w^{2}} / \overline{v^{2}} \approx 1$ extends approximately from $\alpha=0$ to a value of $\alpha$ between 0 and 1 . This value increases from $\approx 0.2$ at low $\beta$ to $\approx 0.8$ at high $\beta$. Outside this range of $\alpha, \overline{w^{2}} / \overline{v^{2}}$ is always lower than 1. For high $\beta$, there is a region, near $\alpha=0.3$, where $\overline{w^{2}} / \overline{v^{2}}>1$. This behaviour is not characteristic of pure streaky structures nor of pure 
streamwise vortices. It can be seen that the maximum values of $\overline{v^{2}} / \overline{u^{2}}$ occur at high $\beta$ for $\alpha=0$. The region where $\overline{v^{2}} / \overline{u^{2}}$ is smaller than one ranges from above $\alpha \approx 0.8$ at low $\beta$ to above $\alpha \approx 0.4$ at higher $\beta . \overline{v^{2}} / \overline{u^{2}}$ has a minimum for $\alpha \approx 0.8$ at high $\beta$. For $\alpha<0$ or $\alpha>1, \overline{w^{2}} / \overline{u^{2}}, \overline{w^{2}} / \overline{v^{2}}$ and $\overline{v^{2}} / \overline{u^{2}}$ do not take very high or low values, remaining near one. The best criterion for distinguishing shear turbulence from Langmuir turbulence is probably that based on $\overline{w^{2}} / \overline{u^{2}}$, because it is approximately independent of $\beta$, as shown by Fig. 3a.

These graphs clearly illustrate that the unstable perturbations that satisfy Craik and Leibovich's instability condition have a complex structure that varies continuously from $\alpha=0$ to $\alpha=1$. The turbulence goes from a state dominated by streamwise vortices (at $\alpha=0$ ) to a state dominated by streaky structures (at $\alpha=1$ ), with an intermediate type of structure in between. At $\alpha=0.5$ and large $\beta$, for example, $\overline{u^{2}}$ and $\overline{w^{2}}$ are similar in magnitude, and both somewhat larger than $\overline{v^{2}}$, and the turbulence is more isotropic than in either extreme case. Presumably, both streamwise vortices and streaky structures exist in this parameter regime. Perhaps these streaky structures existing alongside the streamwise vortices can be identified with the surface jets that have been observed at the confluence zones of the vortices in Langmuir circulations (Leibovich, 1983).

Fig. 3d shows the ratio of minus the shear stress to the TKE, $-\overline{u w} / K$. This ratio, which has been evaluated by Townsend $(1976)$ to be $\approx 0.25$ for shear flows, is slightly smaller for $\alpha=0$ than for $\alpha=1$, and when $0<\alpha<1$ takes considerably higher values. Additionally, when $\alpha=0$ or $\alpha=1$ then $-\overline{u w} / K$ first increases with $\beta$, attains a maximum for some value 
of this parameter, and finally decreases, while for $0<\alpha<1$ it increases monotonically. For $\alpha<0$ or $\alpha>1$, this ratio is small and negative, due to the fact that $\overline{u w}$ is small and positive.

\subsection{Integral length scales}

Additional information about the structure of the turbulence can be obtained by calculating the integral length scales of the velocity fluctuations. These are shown in Fig. 4, normalised by the initial longitudinal integral length scale, $l$. Since the turbulence is initially isotropic, all longitudinal length scales depart from a normalised value of 1 , while the transverse length scales depart from a normalised value of 0.5.

Figs. 4a,b show, respectively, the integral length scales along $x$ and along $y$ of the $u$ velocity fluctuations, $L_{11}^{x}$ and $L_{11}^{y}$. It can be seen that, if $0<\alpha<1$, then $L_{11}^{x}$ increases considerably with $\beta$ while remaining with a value not very different from one for $\alpha \leq 0$ or $\alpha \geq 1$. In contrast, $L_{11}^{y}$ decreases as $\beta$ increases for $0<\alpha \leq 1$, but does not change in magnitude very much otherwise. A somewhat similar behaviour, with some differences, can be observed for $L_{22}^{x}$ and $L_{22}^{y}$ in Figs. 4c,d and for $L_{33}^{x}$ and $L_{33}^{y}$ in Figs. 4e,f. The main differences are that $L_{22}^{x}$ increases with $\beta$ somewhat more slowly than $L_{11}^{x}$ and decreases instead for $\alpha=1$, and that $L_{33}^{x}$ does not increase with $\beta$ for $\alpha=1$. Additionally, $L_{33}^{y}$ decreases for $\alpha=0$ but not for $\alpha=1$. These subtle differences have important consequences for the distinct regimes of streamwise elongation of the turbulence structures. This elongation is borne out more clearly by plotting ratios of the integral length scales along $x$ and along $y$, as is done in Fig. 5 . 


\subsection{Streamwise elongation}

Figs. 5a-c present graphs of the ratios $L_{11}^{x} / L_{11}^{y}, L_{22}^{x} / L_{22}^{y}$ and $L_{33}^{x} / L_{33}^{y}$. These ratios quantify, respectively, the elongation of the $u, v$ and $w$ turbulent velocity structure in an $x-y$ cross-section.

It can be seen that $L_{11}^{x} / L_{11}^{y}, L_{22}^{x} / L_{22}^{y}$ and $L_{33}^{x} / L_{33}^{y}$ grow as $\beta$ increases for $0<\alpha<1$, but do not grow appreciably for $\alpha<0$ or $\alpha>1$. However, there are some subtle differences. For example, $L_{11}^{x} / L_{11}^{y}$ retains exactly its initial value for $\alpha=0$ while it grows for $\alpha=1$. This is a manifestation of the streamwise elongation of the streaky structures that exist in shear flow (cf. Lee et al., 1990). No such elongation of the $u$ velocity fluctuations exists for distortion by an irrotational Stokes drift $(\alpha=0)$. On the other hand, both $L_{22}^{x} / L_{22}^{y}$ and $L_{33}^{x} / L_{33}^{y}$ grow for $\alpha=0$, while not for $\alpha=1$. In particular, $L_{33}^{x} / L_{33}^{y}$ retains exactly its initial value for $\alpha=1$. This is a manifestation of the elongation of the streamwise vortices existing in turbulence distorted by a Stokes drift, while no such elongation exists for distortion by shear $(\alpha=1)$. $L_{11}^{x} / L_{11}^{y}, L_{22}^{x} / L_{22}^{y}$ and $L_{33}^{x} / L_{33}^{y}$ all have a maximum growth rate near $\alpha=0.5$, which indicates that the largest elongation of the turbulence structures in the streamwise direction clearly occurs when both shear and a Stokes drift gradient with the same sign are present. Such elongation exists, in this situation, in all components of the velocity fluctuations, which is consistent with Craik and Leibovich's idea of seeking unstable disturbances for which $k_{1}=0$ (Leibovich, 1977).

\subsection{Physical interpretation of the results}

Following Teixeira and Belcher (2002), the evolution of the turbulent velocity variances can be interpreted in terms of vorticity dynamics (see also 
Teixeira and Belcher, 2010). The simplest case is turbulence distorted by waves $(\alpha=0)$, where the vertical vorticity of the turbulence is simply tilted by the Stokes drift of the waves and amplified as streamwise vorticity. This one-way interaction leads to the amplification of the spanwise $(v)$ and vertical $(w)$ velocity fluctuations, causing the appearance of streamwise vortices.

In the case of a pure shear flow $(\alpha=1)$, there are two sources of vorticity: the shear and the turbulence. In a two-way interaction, the shear tilts the turbulent vorticity in the same way as a Stokes drift, but the turbulent velocity tilts the mean spanwise vorticity $d U / d z$ into vertical and streamwise vorticity (Teixeira and Belcher, 2002, Fig. 15). The streamwise vorticities generated through these two processes partly cancel, so the formation of streamwise vortices is prevented, and streaky structures appear instead, where the streamwise velocity fluctuations $(u)$ are dominant.

In intermediate cases, when shear and a Stokes drift gradient with the same sign distort the turbulence, there are still two sources of vorticity, but the shear constitutes a smaller fraction of the total strain than in the previous case. So the cancellation of streamwise mean and turbulent vorticity does not occur, and streamwise vortices, as well as streaky structures, should be generated. The absence of vorticity cancellation also leads to an instability characterised by exponential growth of the TKE (as will be shown more explicitly next). When the shear and the Stokes drift gradient have different signs, this interpretation based on vorticity dynamics appears not to be as simple, but it is clear that there must occur a certain deal of cancellation of the two strains.

The dynamics of the turbulence can be better understood if one analyses 
the equations giving the evolution of the TKE and of the shear stress. The TKE equation can be derived by multiplying the momentum equation (1) by $\mathbf{u}$ and ensemble averaging. The result is

$$
\frac{\partial K}{\partial t}+\mathbf{U}_{L} \cdot \nabla K=-\overline{u w} \frac{d U_{L}}{d z}-\frac{1}{\rho} \nabla \cdot(\overline{p \mathbf{u}}) .
$$

Dissipation is omitted because it is neglected in the rapid-distortion approach used here. If it is further noted that the turbulence is assumed to be approximately homogeneous, the turbulent correlations and variances do not depend appreciably on position, and their spatial gradients are approximately zero. Therefore, subject to the assumptions of the present model, (17) reduces to

$$
\frac{d K}{d t}=-\overline{u w} \frac{d U_{L}}{d z} .
$$

Hence the production of TKE by shear and by straining by the Stokes drift (both contained in $d U_{L} / d z$ ) is the only reason why the TKE evolves in time.

In order to understand this evolution, it is useful to derive an equation for the shear stress. This can be obtained by multiplying the vertical component of (1) by $u$, adding it to the $x$ component of (1) multiplied by $w$, ensemble averaging and rearranging. This yields

$$
\frac{d \overline{u w}}{d t}=-\overline{w^{2}} \frac{d U}{d z}-\overline{u^{2}} \frac{d U_{S}}{d z}-\frac{1}{\rho}\left(\overline{w \frac{\partial p}{\partial x}}+\overline{u \frac{\partial p}{\partial z}}\right) .
$$

In its present form, this equation is of little use because the time dependence of $\overline{u^{2}}$ and $\overline{w^{2}}$ is unknown. To circumvent this problem, equations may be derived for the evolution of these two quantities, using again the momentum equation, (1):

$$
\left.\begin{array}{l}
\frac{d \overline{u^{2}}}{d t}=-2 \overline{u w} \frac{d U}{d z}-\frac{2}{\rho} \overline{u \frac{\partial p}{\partial x}}, \\
\frac{d \bar{w}^{2}}{d t}=-2 \overline{u w} \frac{d U_{S}}{d z}-\frac{2}{\rho} w \frac{\partial p}{\partial z} .
\end{array}\right\}
$$


When (19) is differentiated with respect to time, and (20) is substituted in it, the following second-order differential equation is obtained:

$$
\frac{d^{2} \overline{u w}}{d t^{2}}-4 \frac{d U}{d z} \frac{d U_{S}}{d z} \overline{u w}=-\frac{1}{\rho} \frac{d}{d t}\left(\overline{w \frac{\partial p}{\partial x}}+\overline{u \frac{\partial p}{\partial z}}\right)+\frac{2}{\rho}\left(\frac{d U}{d z} \overline{w \frac{\partial p}{\partial z}}+\frac{d U_{S}}{d z} \overline{u \frac{\partial p}{\partial x}}\right) .
$$

In this equation it is not possible to neglect the correlations involving the pressure for the reasons invoked in (17), since the pressure terms on the right-hand side cannot be isolated as spatial derivatives of correlations. Nevertheless, these terms are sometimes neglected (e.g. Cambon et al., 1994; Leblanc and Cambon, 1997), because it is assumed that the pressure-strain terms are less important than the production terms: this is sometimes called 'pressure-less dynamics'. In this approximation, which is akin to a 'parcel method', the streamwise velocity perturbation $v$ plays no role, because its kinetic energy is only gained through the pressure-strain terms (see Appendix of Teixeira and Belcher, 2010). Then (21) becomes approximately

$$
\frac{d^{2} \overline{u w}}{d t^{2}}-4 \frac{d U}{d z} \frac{d U_{S}}{d z} \overline{u w} \approx 0
$$

From this simplified form, which is expressed only in terms of $\overline{u w}$, it is possible to infer different types of qualitative behaviour for the shear stress.

When $d U / d z$ and $d U_{S} / d z$ have the same sign, $\overline{u w}$ is the sum of two exponential terms: one growing in time and the other decaying. When $d U / d z$ and $d U_{S} / d z$ have opposite signs, $\overline{u w}$ is the sum of a sine and a cosine and therefore has oscillating behaviour. When either $d U / d z$ or $d U_{S} / d z$ is equal to zero, (22) is singular. Since, in these circumstances, $d^{2} \overline{u w} / d t^{2}=0, \overline{u w}$ can either grow linearly or be a constant. Although the behaviour of the turbulence is not exactly as described because of the neglected pressure terms, 
it is roughly correct, as Fig. 2 corroborates. An approach similar to the present one was used by Teixeira and Belcher (2010) to analyse the turbulent vorticity equation (see their Appendix), and their conclusions have many points in common with those of the present analysis.

It is noteworthy that the condition for exponential growth of the shear stress, $(d U / d z)\left(d U_{S} / d z\right)>0$, is exactly that proposed by Leibovich (1977) for the instability of inviscid neutrally stratified flow to Langmuir circulations. The difference is that this condition was not derived here for the growth of an infinitesimal flow disturbance. The growth rate $2\left[(d U / d z)\left(d U_{S} / d z\right)\right]^{1 / 2}$ is also in accordance with the theory of Leibovich (1977) for inviscid and unstratified conditions. In the present notation, this growth rate can be written $2(\alpha(1-\alpha))^{1 / 2} d U_{L} / d z$. Hence the shear stress should grow by a factor of 2 in a time interval $\left\{\ln 2 /\left[2(\alpha(1-\alpha))^{1 / 2}\right]\right\}$, expressed in units of $\beta$. For $\alpha=0.5$, this time interval has the value $\approx 0.69$. It may be checked in Fig. 2e that this value roughly agrees with the results of the full model, especially for large $\beta$. Nevertheless, neglecting the pressure leads to a slight overestimate of the growth rate. Through the TKE equation, (18), it follows that the TKE must also grow approximately exponentially in this case, and at the same rate as the shear stress. This is indeed supported by Fig. 2d.

By plotting the evolution of the shear stress for $\alpha=0$ and $\alpha=1$ (not shown), it may be verified that $\overline{u w}$ tends to become approximately constant in the first case, while it tends to grow approximately linearly in the second. This implies a faster (approximately quadratic) growth of the TKE with $\beta$ for $\alpha=1$ and an approximately linear growth of the TKE for $\alpha=0$. Obviously, these two cases were outside the scope of the linear stability analyses of Craik 
and Leibovich.

According to $(22)$, when $(d U / d z)\left(d U_{S} / d z\right)<0$, the shear stress and the TKE should undergo oscillations of angular frequency $2\left[-(d U / d z)\left(d U_{S} / d z\right)^{1 / 2}\right]$ or, in the present notation, $2(\alpha(\alpha-1))^{1 / 2} d U_{L} / d z$. In dimensionless units, the period of this oscillation is $\pi /(\alpha(\alpha-1))^{1 / 2}$. These oscillations also reflect on the evolution of the integral length scales and are most easily visible in Fig. 4e, displaying $L_{33}^{x} / l$. For $\alpha=-1$ or $\alpha=2$, for example, the period expressed in units of $\beta$ should be $\pi / \sqrt{2} \approx 2.2$. This is roughly in agreement with the distance that can be observed in that figure between adjacent crests or troughs of the oscillation, but, again, differences are expectable due to neglecting the pressure. In this parameter regime, the TKE not only oscillates but also decays, because the shear stress is small and positive. This presumably happens due to the neglected pressure effects. The consequence of having negative TKE production is that the dissipation rate of TKE (not considered here) must be very small in this case.

\subsection{Analogy with turbulence in a rotating shear flow}

It is worth noting at this point an interesting connection that exists between the model problem treated in this study and problems addressed by previous authors. With the aid of the differential equality

$$
\mathbf{U}_{S} \times \omega=\nabla\left(\mathbf{U}_{S} \cdot \mathbf{u}\right)+\left(\nabla \times \mathbf{U}_{S}\right) \times \mathbf{u}-\left(\mathbf{U}_{S} \cdot \nabla\right) \mathbf{u}-(\mathbf{u} \cdot \nabla) \mathbf{U}_{S},
$$

the momentum equation (1) can be expressed in the following alternative form:

$$
\frac{\partial \mathbf{u}}{\partial t}+\left(\mathbf{U}_{L} \cdot \nabla\right) \mathbf{u}+(\mathbf{u} \cdot \nabla) \mathbf{U}_{L}+2 \boldsymbol{\Omega} \times \mathbf{u}=-\frac{1}{\rho} \nabla p
$$


where $p$ is the uncorrected pressure and

$$
\boldsymbol{\Omega}=-\frac{1}{2} \nabla \times \mathbf{U}_{S}
$$

Equation (24) is a momentum equation with a Coriolis force, where the angular velocity is defined by (25). Hence the momentum equation including a vortex force is equivalent to a momentum equation including the effect of rotation, as long as the Eulerian mean velocity $\mathbf{U}$ is replaced by the total Lagrangian mean velocity $\mathbf{U}_{L}$ and the Coriolis force is defined appropriately in terms of the Stokes drift.

The connection between the effects of streamline curvature and rotation implied by (25) has been noted before, for example by Bradshaw (1969) or Craik (1982), but to the author's knowledge the formal equivalence of the instability conditions in a flow subject to shear and rotation and in a flow subject to shear and Stokes drift has not been established.

For the first type of instability, Bradshaw (1969) defined a criterion, later used and reformulated in clearer terms by Cambon et al. (1994) and Leblanc and Cambon (1997), which states that a flow with shear $S=d U / d z$ and rotation $\Omega$ is unstable to disturbances if

$$
B=\frac{2 \Omega(2 \Omega+S)}{S^{2}}=R(R+1)<0,
$$

where $R=2 \Omega / S$. Replacing in (26) the definition of $\Omega$ given by (25), replacing $S$ by $d U_{L} / d z$, in accordance with (24), and noting also that, in the RDT model used here $d U_{L} / d z=d U / d z+d U_{S} / d z$, the instability condition (26) transposed to a flow described by (24) becomes

$$
\frac{\left(d U_{S} / d z\right)(d U / d z)}{\left(d U_{L} / d z\right)^{2}}=\alpha(1-\alpha)>0,
$$


which is Craik and Leibovich's well-known condition for the instability to Langmuir circulations in the neutrally stratified, inviscid case (Leibovich, 1977). The second equality is again consistent with the fact that, in the turbulent flow under consideration here, the TKE increases strongly for values of $\alpha$ between 0 and 1 .

These results show that the instability of a rotating shear flow and the instability to Langmuir circulations are formally the same. As briefly noted by Craik (1982) and Phillips et al. (1996), the effects of shear and rotation and the effects of shear and Stokes drift are related because the Stokes drift gradient rotates the fluid parcels as they are transported differentially. It is an effect analogous to that of flow with curved streamlines, where the concave curvature (relative to the part of the fluid being studied) is dominant. This happens because the fluid spends more time near the concave surfaces (surface wave crests) than near the convex surfaces (wave troughs), since the fluid speed relative to the wave shape is lower there. Hence the mechanism leading to the formation of streamwise vortices near undulating boundaries is similar to the mechanism responsible for the growth of Taylor-Görtler vortices. In (25) the 'vorticity' is equal to minus the vorticity the Stokes drift would have if it was an Eulerian flow, because the Stokes drift contributes to the total strain but is irrotational.

\subsection{Comparison with data}

As a quick preliminary check on the correctness of the RDT calculations presented in the previous section, comparisons are carried out with the inviscid RDT results of Cambon et al. (1994) and Salhi and Cambon (1997) of turbulence in a rotating shear flow. It is easily shown that the parameter $R$ 
used in these calculations, and introduced above in Section 3.6, corresponds in the present model to $\alpha-1$. So in the following results, for a given value of $R$ used by these previous authors, $\alpha=R+1$ will be used in the present model.

Fig. 6a shows the TKE normalised by its initial value $K_{0}$ as a function of $\beta$ (corresponding to St in Cambon et al. (1994) and Salhi and Cambon (1997)), for various values of $R$ or $\alpha$. Fig. 6b shows the same quantity as a function of $\alpha$ or $R$ for $\beta=5$ (or equivalently $S t=5$ ). Finally, in Fig. 6c the ratio of the integral length scales in the streamwise and spanwise directions is shown as a function of $\beta$ (or $S t$ ) for various values of $R$ or $\alpha$. With the relation between $\alpha$ and $R$ defined above, it can be seen that the agreement is almost perfect, suggesting that the calculations are correct and numerically accurate (small discrepancies could be attributed to an imperfect data extraction from the graphs of Cambon et al. (1994) and Salhi and Cambon (1997)).

More geophysically relevant situations will now be considered. Qualitative support for the turbulence structure described in the previous section is provided, for example, by the laboratory study of Thais and Magnaudet (1996). These authors report that the turbulence beneath surface waves sheared by the wind is characterised by much higher TKE levels than turbulence near solid (flat) walls. Their measurements of the wave-induced shear stress also show that $\overline{u w}$ is highly enhanced near the surface. These results are consistent with the behaviour of the present model in the parameter range $0<\alpha<1$ (which is applicable to wind waves), and is due to the enhancement of TKE production by the shear stress, when the shear and the Stokes drift gradient have the same sign (see Figs. 2d and 2e). 
Thais and Magnaudet (1996) also note that the ratio of the horizontal to the vertical velocity variances is considerably smaller in turbulence beneath surface waves than in the turbulence near a flat wall. For example, in their experiments of wind generated waves, the quantity $\left(\overline{u^{2}} / \overline{w^{2}}\right)^{1 / 2}$ averaged along the vertical direction takes a value of 1.2 , which is smaller than the value quoted as typical of flat-wall boundary layers (1.7). In their experiments of mechanically generated waves, $\left(\overline{u^{2}} / \overline{w^{2}}\right)^{1 / 2}=0.9$, which is even smaller. According to Fig. 3a, this behaviour is again consistent with flow in the parameter range $0<\alpha<1$, since in these conditions $\overline{w^{2}} / \overline{u^{2}}$ is higher than when $\alpha=1$ (pure shear). From the model, it can also be understood why mechanically generated waves have a higher $\overline{w^{2}} / \overline{u^{2}}$ : they are certainly closer to $\alpha=0$, the regime of pure distortion by a Stokes drift, since the shear current induced by the wind is in that case weaker.

Perhaps surprisingly, the experiments of airflow over sinusoidal hills of Gong et al. (1996), and the measurements of flow over complex terrain of Mason and King (1984) and Founda et al. (1997) show essentially similar features. The turbulence measured by these authors is more isotropic and less 'one-dimensional', than in flow over flat terrain, displaying also higher values of $\overline{v^{2}} / \overline{u^{2}}$ and $\overline{w^{2}} / \overline{u^{2}}$. Again, these features are consistent with distortion of the turbulence by a shear and a Stokes drift with the same sign (i.e. $0<\alpha<1$ ). Indeed, the flow over an undulating surface is subject to essentially the same type of distortions as flow beneath surface waves (Phillips et al., 1996), but the situation is considerably more complicated because of the no-slip boundary condition at the surface and of the fact that the 'Stokes drift' in the airflow is strongly rotational (Phillips, 2001). A detailed analysis 
of such situations is outside the scope of the present study.

Various authors (D'Asaro, 2001; Noh et al., 2004) have stressed that the level of vertical TKE is considerably higher in the ocean and in LES of oceanic flows than near a flat boundary. This was one of the motivations for the study of Li et al. (2005), who carried out an extensive exploration of the parameter space using LES of oceanic boundary layers driven by shear, by Stokes drift and by convection. Perhaps the result of Li et al. (2005) that is most amenable to a quantitative comparison with the present model is that shown in their Fig. 5, which plots $\overline{u^{2}} / u_{*}^{2}, \overline{v^{2}} / u_{*}^{2}$ and $\overline{w^{2}} / u_{*}^{2}$ as a function of the turbulent Langmuir number, defined as $L a_{t}=\left(u_{*} / U_{S 0}\right)^{1 / 2}$, where $u_{*}$ is the friction velocity and $U_{S 0}$ is the surface Stokes drift velocity.

Before a comparison can be attempted, it is however necessary to define a relation between the parameter $\alpha$ of the present model and $L a_{t}$. Following Teixeira and Belcher (2010), it may noted that, for a monochromatic surface wave (such as used by Li et al. (2005) to evaluate the Stokes drift)

$$
\frac{d U_{S}}{d z}=2\left(a_{w} k_{w}\right)^{2} \sigma_{w} \mathrm{e}^{-2 k_{w}|z|}
$$

where $a_{w}, k_{w}, \sigma_{w}$ are, respectively, the amplitude, the wavenumber and the angular frequency of the wave and $z$ is the depth. Additionally, assuming that the shear flow in the water has an approximately logarithmic variation,

$$
\frac{d U}{d z}=\frac{u_{* s}}{\kappa|z|}
$$

where $\kappa$ is Von Kármán's constant and $u_{* s}$ is the friction velocity attributable only to the shear flow (cf. Teixeira and Belcher, 2010). The total friction velocity may be defined as $u_{*}^{2}=u_{* s}^{2}+u_{* w}^{2}$, where $u_{* s}$ is a component due to shear and $u_{* w}$ is a component due to the waves. This decomposition is 
justified by the fact, corroborated by Teixeira et al. (2002), that a Stokes drift in the absence of shear is sufficient to generate a shear stress in turbulence subjected to distortion by its vertical gradient. These two components of the friction velocity are parameterised here inspired by the form of (19), as

$$
\begin{aligned}
& u_{* s}^{2}=u_{*}^{2} \frac{\overline{w^{2}}(d U / d z)}{\overline{w^{2}}(d U / d z)+\overline{u^{2}}\left(d U_{S} / d z\right)}=u_{*}^{2} \frac{\overline{w^{2}} \alpha}{\overline{w^{2}} \alpha+\overline{u^{2}}(1-\alpha)} \\
& u_{* w}^{2}=u_{*}^{2} \frac{\overline{u^{2}}\left(d U_{S} / d z\right)}{\overline{w^{2}}(d U / d z)+\overline{u^{2}}\left(d U_{S} / d z\right)}=u_{*}^{2} \frac{\overline{u^{2}}(1-\alpha)}{\overline{w^{2}} \alpha+\overline{u^{2}}(1-\alpha)},
\end{aligned}
$$

(where $-u_{*}^{2}$ is identified with the shear stress). It is conceived that the shear stress will behave essentially as its time derivative concerning the partition of its generation by shear and by the Stokes drift gradient. Equation (30) has the desirable property that $u_{* s}=0$ when $d U / d z=0$ and $u_{* w}=0$ when $d U_{S} / d z=0$. Introducing the first definition of (30) into (29), the ratio of (28) and (29) becomes

$$
\frac{d U_{S} / d z}{d U / d z}=\frac{1-\alpha}{\alpha}=\frac{2 \kappa k_{w}|z| U_{S 0} \mathrm{e}^{-2 k_{w}|z|}}{u_{*}}\left(1+\frac{\overline{u^{2}}}{\overline{w^{2}}} \frac{1-\alpha}{\alpha}\right)^{1 / 2}
$$

where the surface Stokes drift velocity is defined as

$$
U_{S 0}=\left(a_{w} k_{w}\right)^{2} c_{w}
$$

Considering the definition of the turbulent Langmuir number, (31) can also be expressed as

$$
L a_{t}^{2}=2 \kappa k_{w}|z| \mathrm{e}^{-2 k_{w}|z|} \frac{\alpha}{1-\alpha}\left(1+\frac{\overline{u^{2}}}{\overline{w^{2}}} \frac{1-\alpha}{\alpha}\right)^{1 / 2} .
$$

For constant $\alpha$, this expression has a maximum at $k_{w}|z|=1 / 2$. For a fixed value of $L a_{t}$, this corresponds to a minimum of $\alpha$, here called $\alpha_{\min }$ (cf. 
Teixeira and Belcher, 2010) and, according to Fig. 3a, also to a minimum of $\overline{u^{2}} / \overline{w^{2}}$, here called $\left(\overline{u^{2}} / \overline{w^{2}}\right)_{\min }$. Then it follows that

$$
L a_{t}^{2}=\kappa \mathrm{e}^{-1} \frac{\alpha_{\min }}{1-\alpha_{\min }}\left[1+\left(\frac{\overline{u^{2}}}{\overline{w^{2}}}\right)_{\min } \frac{1-\alpha_{\min }}{\alpha_{\min }}\right]^{1 / 2} .
$$

In Fig. 7a, Fig. 5 of Li et al. (2005) is reproduced. Fig. 7b shows $-\overline{u^{2}} / \overline{u w},-\overline{v^{2}} / \overline{u w}$ and $-\overline{w^{2}} / \overline{u w}$ calculated with the present model and plotted for $\beta=2$ taking $\alpha$ as $\alpha_{\text {min }}$ defined above, as a function of $L a_{t}$ calculated from (34). The qualitative agreement of the two figures is remarkable, with a correct ordering of the velocity variances for shear turbulence and for Langmuir turbulence, and the values of $L a_{t}$ where the curves cross each other predicted with great accuracy. The point where $\overline{u^{2}}=\overline{w^{2}}$, in particular, can be determined easily from (34), since from Fig. 3a, this should correspond roughly to $\alpha_{\min }=1 / 2$ and $\left(\overline{u^{2}} / \overline{w^{2}}\right)_{\min }=1$. If these values are used, one obtains a critical $L a_{t}$ (where the transition from shear turbulence to Langmuir turbulence occurs according to this criterion) given by

$$
\left(L a_{t}\right)_{c r i t}=\left(\sqrt{2} \mathrm{e}^{-1} \kappa\right)^{1 / 2} \text {. }
$$

Using $\kappa=0.4$, this is evaluated to be $\left(L a_{t}\right)_{\text {crit }}=0.456$ and is plotted in Fig. 7 as the vertical dotted line. The agreement of this line with the point where $\overline{w^{2}} / \overline{u^{2}}=1$ both in Fig. $7 \mathrm{~b}$ (where $\alpha=\alpha_{\min }$ everywhere and thus where the best agreement would be expected) and with Fig. 7a (where the conditions are more complex) is remarkable. The vertical dashed line corresponds to the same calculation if in (29) the full friction velocity was considered instead of $u_{* s}$. This would amount to neglecting the factor inside square brackets in (34) and the square-root of 2 in (35), giving instead $\left(L a_{t}\right)_{\text {crit }}=0.384$. 
Clearly, the larger value of $\left(L a_{t}\right)_{c r i t}$ is more accurate than the smaller one, emphasising the importance of partitioning the shear stress as in (30).

In other detailed features, the agreement of Figs. 7a and 7b is less satisfactory. For example, the present model underestimates the increase of $\overline{u^{2}} / u_{*}^{2}$ for low values of $L a_{t}$ and overestimates both $\overline{u^{2}} / u_{*}^{2}$ and $\overline{v^{2}} / u_{*}^{2}$ for high $L a_{t}$. The difference between $\overline{w^{2}}$ and either $\overline{u^{2}}$ or $\overline{v^{2}}$ for high $L a_{t}$ is also too marked (a well-known deficiency of RDT - see Townsend (1976)). But, given the fact that the quantities plotted in Fig. 7a are averaged in the vertical, and that the mean velocity profile varies vertically in a complicated way in the LES of Li et al. (2005), the agreement is surprisingly good.

It is also noteworthy that no blocking effects of the air-water interface on the turbulence have been taken into account in the calculations (cf. Teixeira and Belcher, 2006, 2010). This is not too problematic, because the regions of the flow where these effects are felt correspond to a negligible fraction of the depth intervals used by Li et al. (2005) to compute the averages presented in Fig. 7a. The method for relating $L a_{t}$ to $\alpha$ is, necessarily, one of the most questionable aspects of the procedure outlined above, and obviously many alternatives could be devised.

\section{Conclusions}

The structure of turbulence beneath surface water waves was investigated theoretically using RDT. In the surface layer of the ocean, the turbulence is dynamically affected essentially by two external forcings, which distort its vorticity: the vertical gradient of the mean Eulerian current, and the vertical gradient of the Stokes drift transport, associated with the surface waves. 
The evolution of the turbulence energy, anisotropy and elongation due to these two forcings was studied for initially isotropic turbulence through the calculation of Reynolds stresses and integral length scales, and their ratios.

The parameter space was explored for situations with distortion by shear only, by Stokes drift only (relevant to swell), by shear and a Stokes drift gradient with the same sign (relevant to wind waves), and by shear and a Stokes drift gradient of opposite signs (relevant to waves propagating against the wind). The TKE grows algebraically for distortion by shear only or by Stokes drift only, decays for shear and a Stokes drift gradient of opposite signs, and grows exponentially for shear and a Stokes drift gradient with the same sign. This is a manifestation of the linear instability mechanism investigated by Craik and Leibovich (1976), which is included in the possible modes of growth of disturbances in RDT. Craik and Leibovich's linear instability condition for Langmuir circulations in the inviscid and unstratified limit was found to be formally equivalent to the linear instability condition for a rotating shear flow, investigated by Bradshaw (1969), if the Eulerian mean velocity is replaced by the Lagrangian mean velocity (including the Stokes drift) and if the rotation rate is related to the gradient of the Stokes drift in an appropriate way.

The anisotropy of the turbulence was found to range from that typical of streaky structures (where the streamwise velocity fluctuations are dominant and elongated in the streamwise direction) when only shear exists, to that typical of streamwise vortices (where the spanwise and vertical velocity fluctuations are dominant and elongated in the same direction) when only the Stokes drift exists. When the shear and the Stokes drift gradient have 
the same sign, the turbulence is highly energetic and more isotropic, with all velocity fluctuations strongly elongated in the streamwise direction. It apparently contains both streaky structures and streamwise vortices. On the other hand, when the shear and the Stokes drift gradient have opposite signs, the turbulence is weak, rather isotropic, and not elongated.

The fact that Langmuir circulations have often been observed, or simulated numerically, with a structure close to that of pure streamwise vortices suggests that the effect of the Stokes drift becomes dominant as the circulations evolve. This is consistent with observations by Smith (1998), which show that, in well-developed Langmuir circulations, the friction velocity no longer enters directly in scaling the motion. This behaviour is probably due to the suppression of the shear current by the strong vertical mixing associated with the large vertical velocity variances that exist when $0 \leq \alpha<1$. The experiments of Melville et al. (1998) support this view.

The turbulence structure produced by the present model in the parameter range $0<\alpha<1$ is consistent with the structure of turbulence beneath water waves in the experiments of Thais and Magnaudet (1996), and surprisingly also with the structure of turbulence in atmospheric flows over undulating terrain (Mason and King, 1984; Gong et al., 1996). In all of these cases, no Langmuir circulations as usually defined were reported, but the turbulence was more isotropic and energetic than in flat-wall boundary layers. The comparison carried out with the LES data of Li et al. (2005) for $\overline{u^{2}} / u_{*}^{2}, \overline{v^{2}} / u_{*}^{2}$ and $\overline{w^{2}} / u_{*}^{2}$ as a function of $L a_{t}$ showed that the transition of the anisotropy from shear turbulence to Langmuir turbulence is reproduced qualitatively well by RDT. The value of $L a_{t}$ at which this transition occurs is also predicted 
accurately.

All these results highlight the potential usefulness of RDT for developing physically-based parameterisations of turbulent processes in the oceanic boundary layer.

\section{Acknowledgements}

I acknowledge the financial support of the Portuguese Science Foundation (FCT) under Project AWARE/PTDC/CTE-ATM/65125/2006.

\section{References}

Andrews, D. G., McIntyre, M. E., 1978. An exact theory of nonlinear waves on a Lagrangian-mean flow. Journal of Fluid Mechanics 89, 609-646.

Ardhuin, F., Jenkins, A. D., 2006. On the interaction of surface waves and upper ocean turbulence. Journal of Physical Oceanography 36, 551-557.

Ardhuin, F., Marié, L., Rascle, N., Forget, P., Roland, A., 2009. Observation and estimation of Lagrangian, Stokes, and Eulerian currents induced by wind and waves at the sea surface. Journal of Physical Oceanography 39, $2820-2838$.

Ardhuin, F., Rascle, N., Belibassakis, K. A., 2008. Explicit wave-averaged primitive equations using a generalized Lagrangian mean. Ocean Modelling $20,35-60$.

Batchelor, G. K., Proudman, I., 1954. The effect of rapid distortion of a fluid in turbulent motion. Quarterly Journal of Mechanics and Applied Mathematics 7, 83-103. 
Belcher, S. E., Harris, J. A., Street, R. L., 1994. Linear dynamics of wind waves in coupled turbulent air-water flow. Part 1. Theory. Journal of Fluid Mechanics 271, 119-151.

Bradshaw, P., 1969. The analogy between streamline curvature and buoyancy in turbulent shear flow. Journal of Fluid Mechanics 36, 177-191.

Cambon, C., Benoit, J.-P., Shao, L., Jacquin, L., 1994. Stability analysis and large-eddy simulation of rotating turbulence with organized eddies. Journal of Fluid Mechanics 278, 175-200.

Cambon, C., Scott, J. F., 1999. Linear and nonlinear models of anisotropic turbulence. Annual Review of Fluid Mechanics 31, 1-53.

Craik, A. D. D., 1977. The generation of Langmuir circulations by an instability mechanism. Journal of Fluid Mechanics 81, 209-223.

Craik, A. D. D., 1982. The generalized Lagrangian-mean equations and hydrodynamic stability. Journal of Fluid Mechanics 125, 27-35.

Craik, A. D. D., Leibovich, S., 1976. A rational model for Langmuir circulations. Journal of Fluid Mechanics 73, 401-426.

D'Asaro, E., 2001. Turbulent vertical kinetic energy in the ocean mixed layer. Journal of Physical Oceanography 31, 3530-3537.

Faller, A. J., Auer, S. J., 1988. The roles of Langmuir circulations in the dispersion of surface tracers. Journal of Physical Oceanography 18, 11081123. 
Founda, D., Tombrou, M., Lalas, D. P., Asimakopoulos, D. N., 1997. Some measurements of turbulence characteristics over complex terrain. Boundary Layer Meteorology 83, 221-245.

Gartshore, I. S., Durbin, P. A., Hunt, J. C. R., 1983. The production of turbulent stress in a shear flow by irrotational fluctuations. Journal of Fluid Mechanics 137, 307-329.

Gong, W., Taylor, P. A., Dörnbrack, A., 1996. Turbulent boundary-layer flow over fixed aerodynamically rough two-dimensional sinusoidal waves. Journal of Fluid Mechanics 312, 1-37.

Grant, A. L. M., Belcher, S. E., 2009. Characteristics of Langmuir turbulence in the ocean mixed layer. Journal of Physical Oceanography 39, 1871-1887.

Hunt, J. C. R., 1984. Turbulence structure in thermal convection and shearfree boundary layers. Journal of Fluid Mechanics 138, 161-184.

Hunt, J. C. R., Carruthers, D. J., 1990. Rapid distortion theory and the 'problems' of turbulence. Journal of Fluid Mechanics 212, 497-532.

Kantha, L., Wittmann, P., Sclavo, M., Carniel, S., 2009. A preliminary estimate of the Stokes dissipation of wave energy in the global ocean. Geophysical Research Letters 36, L02605.

Kevlahan, N. K.-R., Hunt, J. C. R., 1997. Nonlinear interactions in turbulence with strong irrotational straining. Journal of Fluid Mechanics 337, $333-364$. 
Kline, S. J., Reynolds, W. C., Shraub, F. A., Runstadler, P. W., 1967. The structure of turbulent boundary layers. Journal of Fluid Mechanics 30, $741-773$.

Leblanc, S., Cambon, C., 1997. On the three-dimensional instabilities of plane flows subjected to Coriolis force. Physics of Fluids 9, 1307-1316.

Lee, M. J., Kim, J., Moin, P., 1990. Structure of turbulence at high shear rate. Journal of Fluid Mechanics 216, 561-583.

Leibovich, S., 1977. Convective instability of stably stratified water in the ocean. Journal of Fluid Mechanics 82, 561-581.

Leibovich, S., 1980. On wave-current interaction theories of Langmuir circulations. Journal of Fluid Mechanics 99, 715-724.

Leibovich, S., 1983. The form and dynamics of Langmuir circulations. Annual Review of Fluid Mechanics 15, 391-427.

Li, M., Garrett, C., Skyllingstad, E., 2005. A regime diagram for classifying turbulent large eddies in the upper ocean. Deep-Sea Research 52, 259-278.

Mann, J., 1994. The spatial structure of neutral atmospheric surface-layer turbulence. Journal of Fluid Mechanics 273, 141-168.

Mason, P. J., King, J. C., 1984. Atmospheric flow over a succession of nearly two-dimensional ridges and valleys. Quarterly Journal of the Royal Meteorological Society 110, 821-845.

McWilliams, J. C., Sullivan, P. P., Moeng, C.-H., 1997. Langmuir turbulence in the ocean. Journal of Fluid Mechanics 334, 1-30. 
Melville, W. K., Shear, R., Veron, F., 1998. Laboratory measurements of the generation and evolution of Langmuir circulations. Journal of Fluid Mechanics 364, 31-58.

Noh, Y., Min, H. S., Raasch, S., 2004. Large eddy simulation of the ocean mixed layer: the effects of wave breaking and Langmuir circulation. Journal of Physical Oceanography 34, 720-735.

Phillips, W. R. C., 2001. On the pseudomomentum and generalized Stokes drift in a spectrum of rotational waves. Journal of Fluid Mechanics 430, 209-229.

Phillips, W. R. C., Wu, Z., 1994. On the instability of wave-catalysed longitudinal vortices in strong shear. Journal of Fluid Mechanics 272, 235-254.

Phillips, W. R. C., Wu, Z., Lumley, J. L., 1996. On the formation of longitudinal vortices in a turbulent boundary layer over wavy terrain. Journal of Fluid Mechanics 326, 321-341.

Polton, J. A., Belcher, S. E., 2007. Langmuir turbulence and deeply penetrating jets in an unstratified mixed layer. Journal of Geophysical Research 112, C09020.

Rascle, N., Ardhuin, F., Terray, E. A., 2006. Drift and mixing under the ocean surface: A coherent one-dimensional description with application to unstratified conditions. Journal of Geophysical Research 111, C03016.

Salhi, A., Cambon, C., 1997. An analysis of rotating shear flow using linear theory and DNS and LES results. Journal of Fluid Mechanics 347, 171-195. 
Savill, A. M., 1987. Recent developments in rapid-distortion theory. Annual Review of Fluid Mechanics 19, 531-575.

Shrira, V. I., 1993. Surface waves on shear currents: solution of the boundaryvalue problem. Journal of Fluid Mechanics 252, 565-584.

Smith, J. A., 1998. Evolution of Langmuir circulation during a storm. Journal of Geophysical Research 103, 12649-12668.

Speziale, C. G., Abid, R., Blaisdell, G. A., 1996. On the consistency of Reynolds stress turbulence closures with hydrodynamic stability theory. Physics of Fluids 8, 781-788.

Speziale, C. G., MacGiolla Mhuiris, N., 1989. On the prediction of equilibrium states in homogeneous turbulence. Journal of Fluid Mechanics 209, $591-615$.

Teixeira, M. A. C., Belcher, S. E., 2000. Dissipation of shear-free turbulence near boundaries. Journal of Fluid Mechanics 422, 167-191.

Teixeira, M. A. C., Belcher, S. E., 2002. On the distortion of turbulence by a progressive surface wave. Journal of Fluid Mechanics 458, 229-267.

Teixeira, M. A. C., Belcher, S. E., 2006. On the initiation of surface waves by turbulent shear flow. Dynamics of Atmospheres and Oceans 41, 1-27.

Teixeira, M. A. C., Belcher, S. E., 2010. On the structure of Langmuir turbulence. Ocean Modelling 31, 105-119.

Thais, L., Magnaudet, J., 1996. Turbulent structure beneath surface gravity waves sheared by the wind. Journal of Fluid Mechanics 328, 313-344. 
Townsend, A. A., 1970. Entrainment and the structure of turbulent flow. Journal of Fluid Mechanics 41, 13-46.

Townsend, A. A., 1976. The structure of turbulent shear flow. Cambridge University Press. 


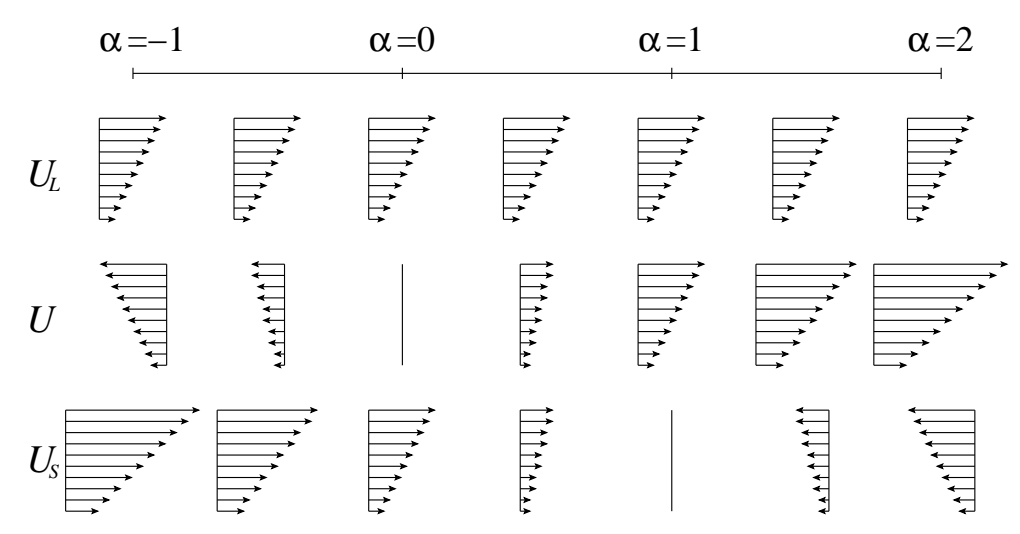

Figure 1: 

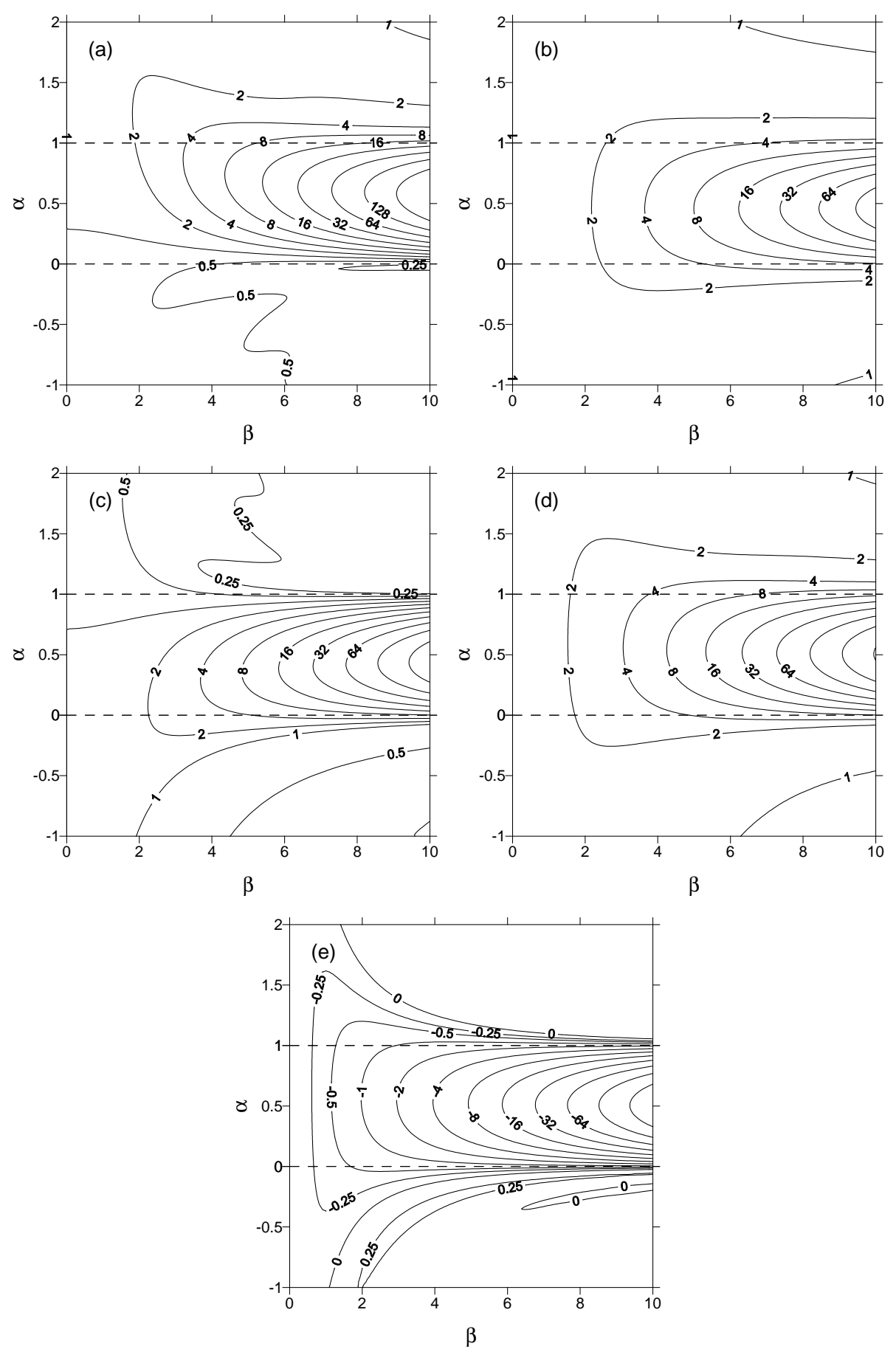

Figure 2: 

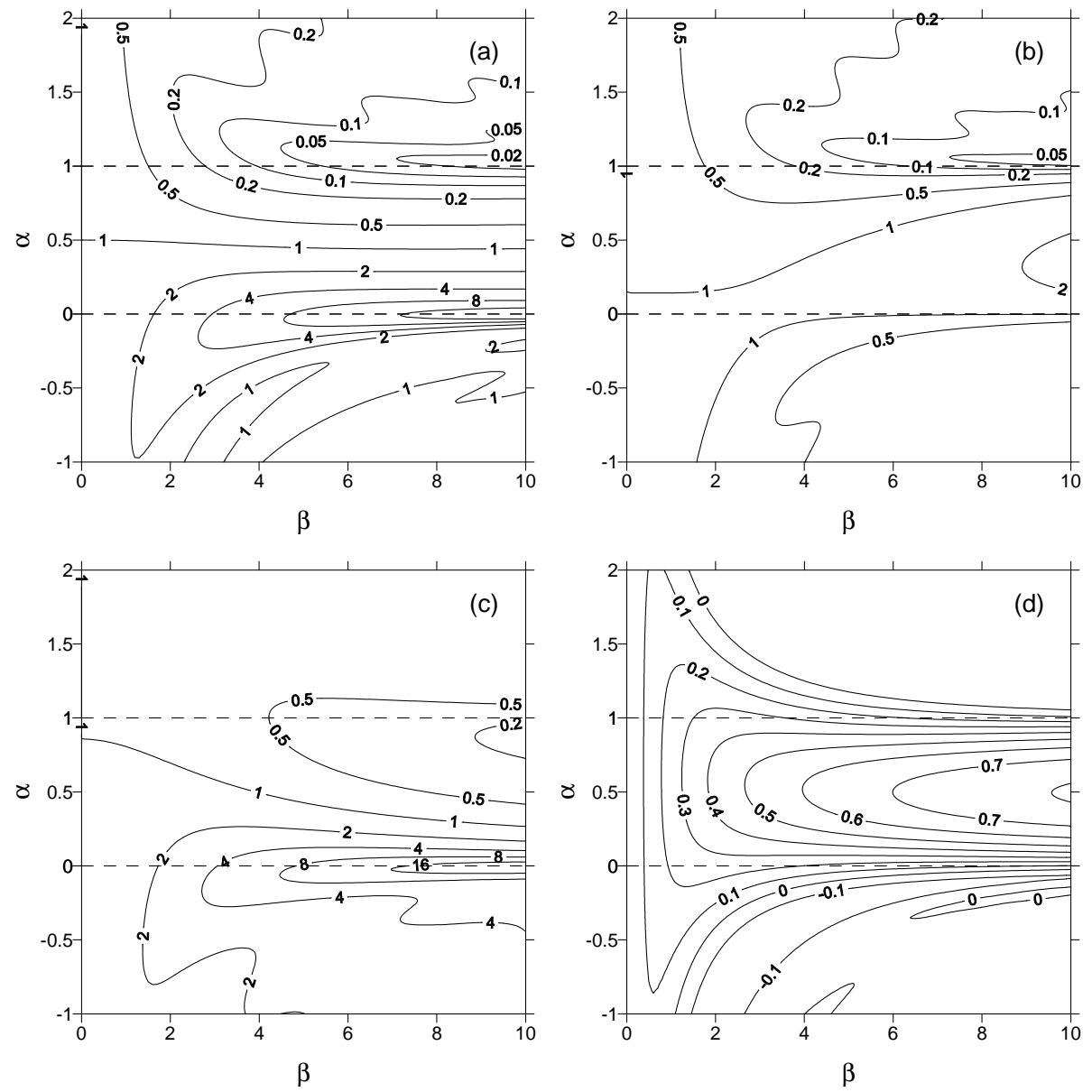

Figure 3: 

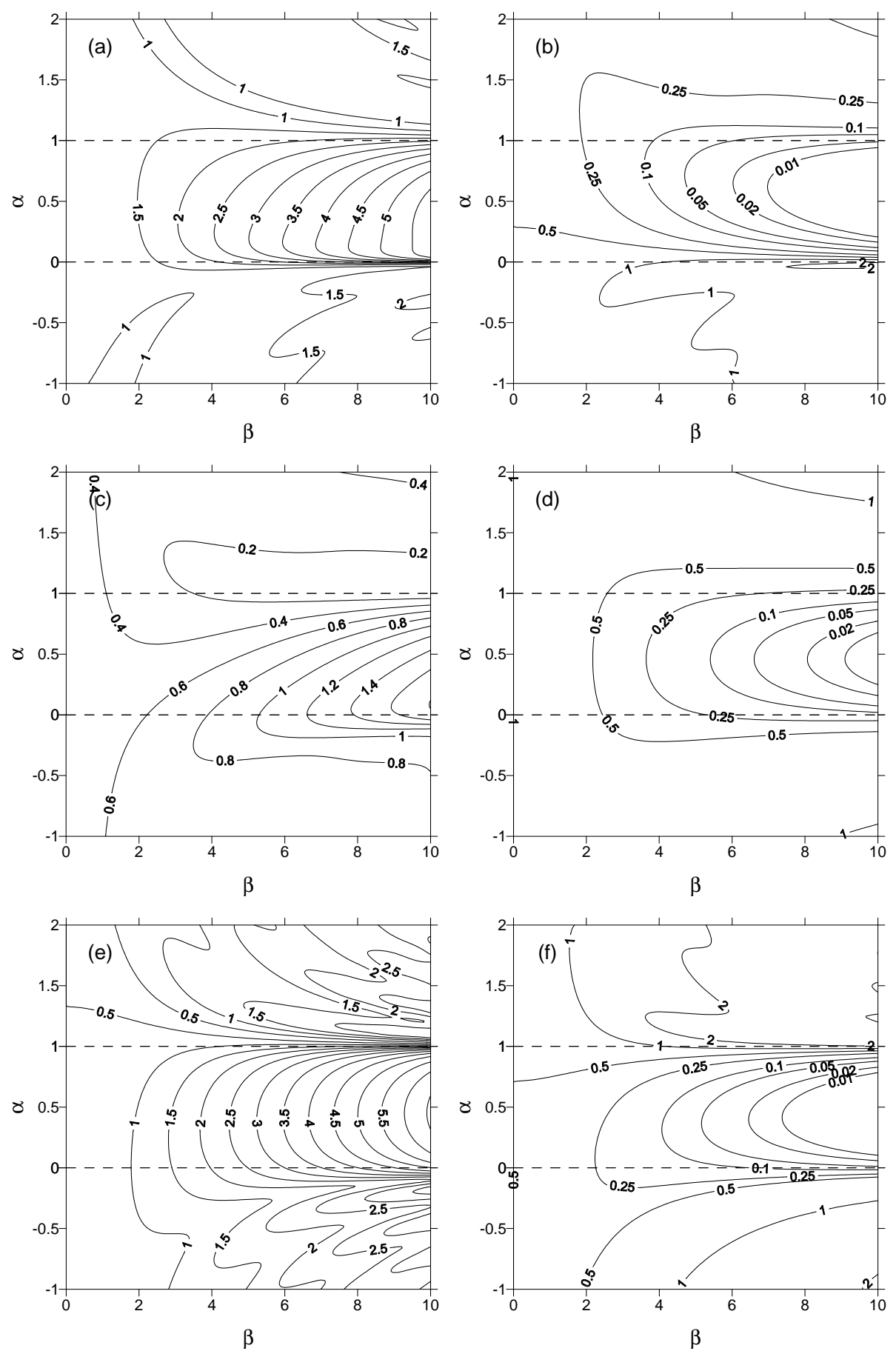

Figure 4: 

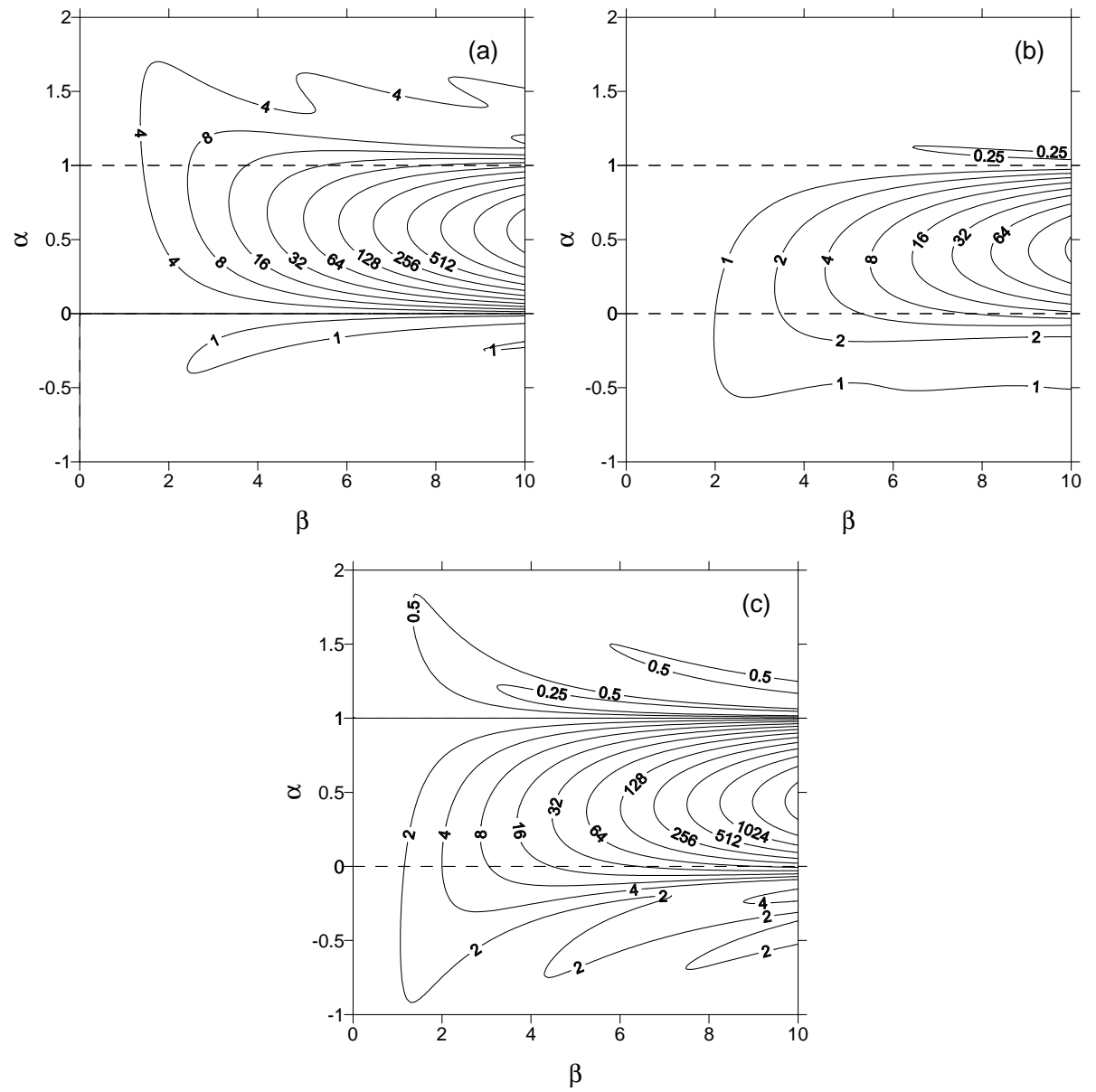

Figure 5: 

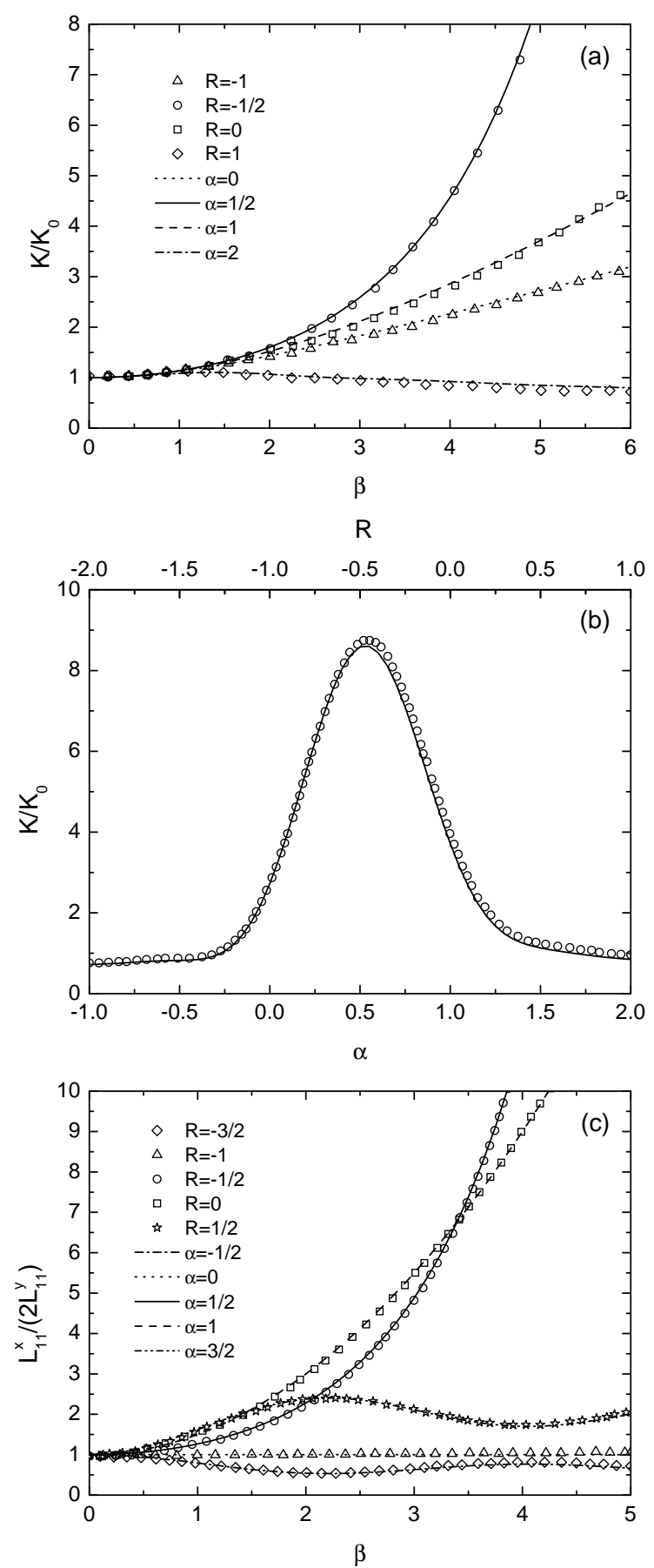

Figure 6: 

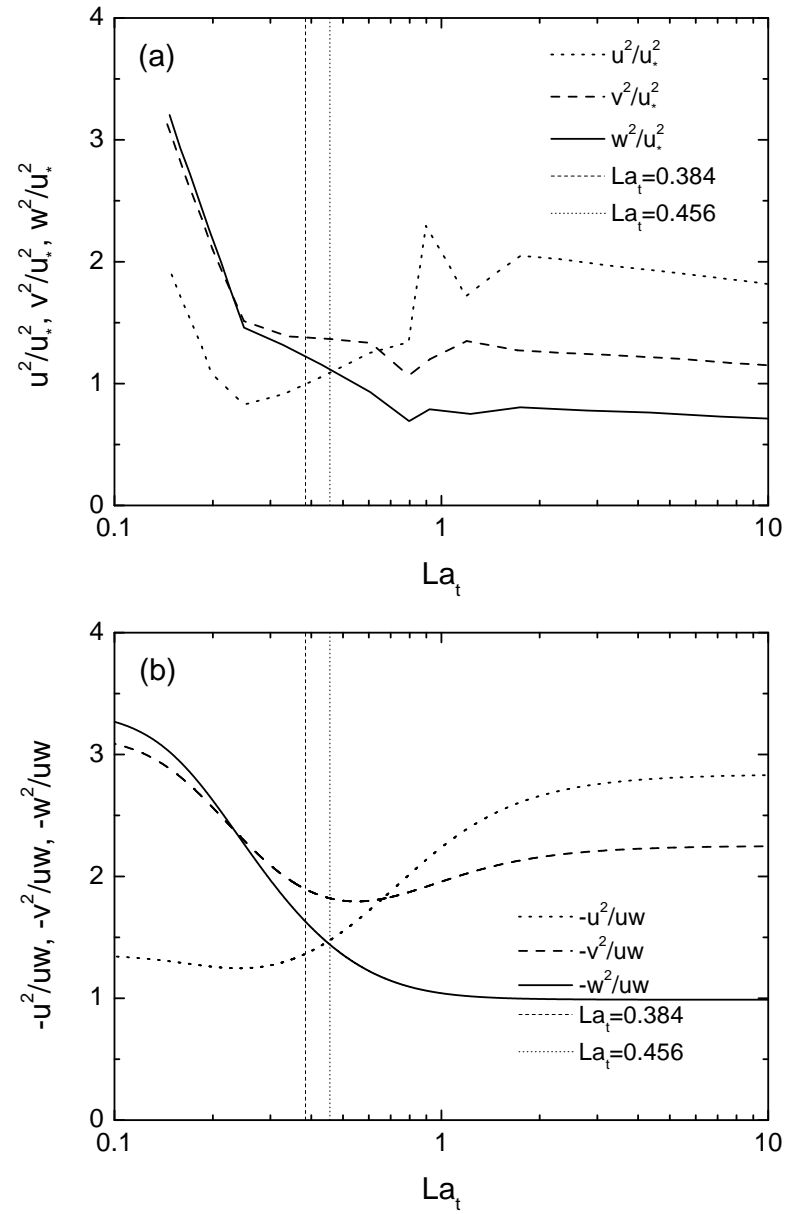

Figure 7: 


\section{Figure captions}

Figure 1: Diagram showing schematic profiles of the vertical gradient of the total Lagrangian transport, $d U_{L} / d z$ (assumed to be constant), of the vertical gradient of the Eulerian velocity, $d U / d z$, and of the vertical gradient of the Stokes drift transport, $d U_{S} / d z$, for the range of $\alpha$ considered in the present study.

Figure 2: Reynolds stresses normalised by the initial RMS velocity, $q$, as a function of $\alpha$ and $\beta$. (a) streamwise velocity variance, $\overline{u^{2}} / q^{2}$, (b) spanwise velocity variance, $\overline{v^{2}} / q^{2}$, (c) vertical velocity variance, $\overline{w^{2}} / q^{2}$, (d) turbulent kinetic energy, $K / q^{2}$, (e) shear stress, $\overline{u w} / q^{2}$.

Figure 3: Ratios of the Reynolds stresses, as a function of $\alpha$ and $\beta$. (a) vertical to streamwise velocity variance, $\overline{w^{2}} / \overline{u^{2}}$, (b) vertical to spanwise velocity variance, $\overline{w^{2}} / \overline{v^{2}}$, (c) spanwise to streamwise velocity variance, $\overline{v^{2}} / \overline{u^{2}}$, (d) minus the shear stress to TKE, $-\overline{u w} / K$.

Figure 4: Integral length scales normalised by the initial longitudinal length scale, $l$, as a function of $\alpha$ and $\beta$. (a) $L_{11}^{x} / l$, (b) $L_{11}^{y} / l$, (c) $L_{22}^{x} / l$, (d) $L_{22}^{y} / l$, (e) $L_{33}^{x} / l$, (f) $L_{33}^{y} / l$.

Figure 5: Ratios of the integral length scales in the streamwise $(x)$ and in the spanwise $(y)$ directions as a function of $\alpha$ and $\beta$. (a) $L_{11}^{x} / L_{11}^{y}$, (b) $L_{22}^{x} / L_{22}^{y}$, (c) $L_{33}^{x} / L_{33}^{y}$.

Figure 6: Comparison between results from the present model (lines) and from inviscid RDT calculations of turbulence in a rotating shear flow by 
Cambon et al. (1994) and Salhi and Cambon (1997) (symbols). (a) Normalised TKE, $K / K_{0}$, as a function of $\beta$. (b) Normalised TKE as a function of $\alpha$ (or $R$ ). (c) $L_{11}^{x} /\left(2 L_{11}^{y}\right)$ as a function of $\beta$.

Figure 7: Velocity variances normalised by the friction velocity $\left(\overline{u^{2}} / u_{*}^{2}, \overline{v^{2}} / u_{*}^{2}\right.$ and $\left.\overline{w^{2}} / u_{*}^{2}\right)$ or by the shear stress $\left(-\overline{u^{2}} / \overline{u w},-\overline{v^{2}} / \overline{u w}\right.$ and $\left.-\overline{w^{2}} / \overline{u w}\right)$ as a function of $L a_{t}$. (a) Results from Fig. 5 of Li et al. (2005). (b) Results from the present model at $\beta=2$ and with $L a_{t}$ estimated from (34). The vertical lines predict the value of $L a_{t}$ at which $\overline{w^{2}} / \overline{u^{2}}=1$. 